\title{
ANALYTICAL INVESTIGATIONS OF THE SUMUDU TRANSFORM AND APPLICATIONS TO INTEGRAL PRODUCTION EQUATIONS
}

\author{
FETHI BIN MUHAMMED BELGACEM, \\ AHMED ABDULLATIF KARABALLI, AND SHYAM L. KALLA
}

Received 16 July 2002 and in revised form 8 October 2002

The Sumudu transform, whose fundamental properties are presented in this paper, is little known and not widely used. However, being the theoretical dual to the Laplace transform, the Sumudu transform rivals it in problem solving. Having scale and unitpreserving properties, the Sumudu transform may be used to solve problems without resorting to a new frequency domain. Here, we use it to solve an integral productiondepreciation problem.

\section{Introduction}

In [6], a new integral transform, called the Sumudu transform defined for functions of exponential order, is proclaimed. We consider functions in the set $A$, defined by

$$
\begin{aligned}
A=\{ & f(t) \mid \exists M, \tau_{1}, \text { and } / \text { or } \tau_{2}>0, \\
& \text { such that } \left.|f(t)|<M e^{|t| / \tau_{j}}, \text { if } t \in(-1)^{j} \times[0, \infty)\right\} .
\end{aligned}
$$

For a given function in the set $A$, the constant $M$ must be finite, while $\tau_{1}$ and $\tau_{2}$ need not simultaneously exist, and each may be infinite. Instead of being used as a power to the exponential as in the case of the Laplace transform, the variable $u$ in the Sumudu transform is used to factor the variable $t$ in the argument of the function $f$. Specifically, for $f(t)$ in $A$, the Sumudu transform is defined by

$$
G(u)=\mathbb{S}[f(t)]= \begin{cases}\int_{0}^{\infty} f(u t) e^{-t} d t, & 0 \leq u<\tau_{2}, \\ \int_{0}^{\infty} f(u t) e^{-t} d t, & -\tau_{1}<u \leq 0 .\end{cases}
$$

Albeit similar in expression, the two parts in the previous definition arise because in the domain of $f$, the variable $t$ may not change sign. For instance, if a function is defined for nonnegative $t$, then $G(u)$ is solely defined for nonnegative $u$, as exemplified in the prototypical case of the function $f(t)=\sqrt{t}$. Here, $\tau_{1}$ is simply not needed, $M$ can be 
taken equal to 1, while $\tau_{2}$ is infinite. Entry 5 in Table A.1 shows that this function maps to a multiple constant of itself by the Sumudu transform. Alternatively, see (1.12). On the other hand, for $u \in(-\infty, 1 / a)$, the Sumudu transform of $e^{a t}$ is

$$
G(u)=\frac{1}{1-a u}
$$

While we are in agreement with most of the claims expounded by Watugala [6], we think that this transform is not so new as proclaimed. The Sumudu transform is connected to the $s$-multiplied Laplace transform (see [5]). This however in no way diminishes its importance or usefulness. In fact, we show that the Sumudu transform has deeper connections with the Laplace transform than previously established. We also present many of the new transform properties that make it uniquely qualified to address and solve some applied problems, especially ones in which the units of the problem must be preserved.

The discrete analog of the Sumudu integral transform (1.2) is defined for power series functions $f(t)=\sum_{k=0}^{\infty} a_{k} t^{k}$, having an interval of convergence containing $t=0$, as follows:

$$
G(u)=\sum_{k=0}^{\infty} k ! a_{k} u^{k} \quad \text { for } u \in\left(-\tau_{1}, \tau_{2}\right) \text {. }
$$

So, the linear function $f(t)=a_{0}+a_{1} t$ transforms to itself, $G(u)=a_{0}+a_{1} u=f(u)$. However, the power series

$$
f(t)=\sum_{k=0}^{\infty}(-1)^{k} \frac{(a t)^{k}}{k !}=e^{-a t}
$$

transforms to the geometric series

$$
G(u)=\sum_{k=0}^{\infty}(-1)^{k}(a u)^{k}=\frac{1}{1+a u},
$$

with $u$ in $(-1 / a, 1 / a)$.

Equations (1.4), (1.5), and (1.6) reveal that the Sumudu transform amplifies the coefficients of the power series according to their order, without changing the initial units of the series. Therefore, a signal with increasingly decaying higher-order coefficients $a_{n}$ transforms to another with much more prominent tail end. So, the power series of $e^{t}$ which converges throughout $\mathbb{R}$ transforms to the geometric series of $1 /(1-t)$ which converges only in the interval $(-1,1)$. Moreover, the discrete version of the Sumudu transform gives us the insight of how to obtain $f(t)$ from $G(u)$. We simply divide the coefficients of the power series for $G(u)$ by the respective $n$ ! value to obtain the power series for $f(t)$.

While it is harder to compute at times, the integral transform in (1.2) is clearly much more general than its discrete counterpart defined in (1.4). May they be of differential, integral, or engineering control nature, the Sumudu transform can certainly treat all problems that are usually treated by the well-known and extensively used Laplace transform 
defined for $\mathfrak{R}(s)>0$ by

$$
F(s)=\mathfrak{E}(f(t))=\int_{0}^{\infty} e^{-s t} f(t) d t
$$

Indeed, as the next theorem shows, the Sumudu transform is closely connected with the Laplace transform.

Theorem 1.1. Let $f(t) \in A$ with Laplace transform $F(s)$. Then the Sumudu transform $G$ of $f(t)$ is given by

$$
G(u)=\frac{F(1 / u)}{u}
$$

Proof. Let $f(t) \in A$, then for $-\tau_{1}<u<\tau_{2}$,

$$
G(u)=\int_{0}^{\infty} e^{-t} f(u t) d t
$$

If we set $w=u t(t=w / u)$, then the right-hand side can be written as

$$
G(u)=\int_{0}^{\infty} e^{-w / u} f(w) \frac{d w}{u}=\frac{1}{u} \int_{0}^{\infty} e^{-w / u} f(w) d w .
$$

The integral on the right-hand side is clearly $F(1 / u)$, thus yielding (1.8).

We observe that $G(1)=F(1)$ so that both the Sumudu and Laplace transforms must coincide at $u=s=1$. Furthermore, since for $x>0$, the Gamma function

$$
\Gamma(x)=\int_{0}^{\infty} t^{x-1} e^{-t} d t
$$

is the Laplace transform of $t^{x-1}\left(\mathfrak{E}\left(t^{x-1}\right)\right)$ when $s=1$, then $\Gamma(x)$ must also be the Sumudu transform $\left(\mathbb{S}\left(t^{x-1}\right)\right)$ when $u=1$. Indeed, multiplying the integral in (1.11) by $u^{x-1}$ yields the following result.

Corollary 1.2. For $x>0$, the Sumudu transform of $t^{x-1}$ is

$$
G(u)=\mathbb{S}\left(t^{x-1}\right)=\Gamma(x) u^{x-1} .
$$

In fact, the connection of the Sumudu transform with the Laplace transform goes much deeper. Therefore, the roles of $F$ and $G$ in (1.8) can be interchanged.

Corollary 1.3. Let $f(t) \in A$, having $F$ and $G$ for Laplace and Sumudu transforms, respectively. Then

$$
F(s)=\frac{G(1 / s)}{s}
$$

Proof. Equation (1.13) can be obtained from (1.8) by taking $u=1 / s$. 
The pair of equations (1.8) and (1.13) forms the duality relation governing these two transforms and may serve as a means to get one from the other when needed. Following the style of Kreyszig [4], Table A.1 shows both Laplace and Sumudu transforms of some elementary and special functions. Table A.2 summarizes the properties of the Sumudu transform as expanded upon below.

\section{Sumudu transforms of derivatives and integrals}

Let $f(t) \in A$ and let $F(s)=\mathfrak{E}(f(t))$, the Laplace transform of $f(t)$ with respect to $s$, and let $G(u)=\mathbb{S}(f(t))$; then

$$
G(u)=\frac{F(1 / u)}{u}
$$

Being a restatement of the duality relation (1.8), (2.1) will serve as our working definition throughout the paper. Since the Laplace transform of $\sin t$ is $1 /\left(1+s^{2}\right)$, then in view of (2.1), its Sumudu transform is

$$
\mathbb{S}[\sin t]=\frac{u}{1+u^{2}},
$$

which is the Laplace transform of $\cos t$ (with $u=s$ ). This exemplifies the duality between these two transforms, and hence emphasizes the importance of the Sumudu transform.

Obviously, the Sumudu transform is linear since the Laplace transform is. The next few theorems are designed to illustrate how the Sumudu transform behaves with derivatives and antiderivatives.

Theorem 2.1. Let $F_{1}(u)$ and $G_{1}(u)$ be the Laplace and the Sumudu transforms of the derivative of $f(t)(\in A)$. Then

$$
G_{1}(u)=\frac{G(u)-f(0)}{u} .
$$

Proof. Since the Laplace transform of the derivative of $f(t)$ is

$$
F_{1}(s)=s F(s)-f(0),
$$

then

$$
G_{1}(u)=\frac{F_{1}(1 / u)}{u}=\frac{F(1 / u) / u-f(0)}{u}
$$

or

$$
G_{1}(u)=\frac{G(u)-f(0)}{u} .
$$

Clearly, from (2.2), being the derivative of $\sin t$, relation (2.3) prescribes the expected Sumudu transform for cost:

$$
\mathbb{S}[\cos t]=\frac{1}{1+u^{2}}
$$


Theorem 2.2. Let $n \geq 1$, and let $G_{n}(u)$ and $F_{n}(u)$ be the Sumudu and Laplace transforms of the nth derivative $f^{(n)}(t)$, of the function $f(t)$, respectively. Then

$$
G_{n}(u)=\frac{G(u)}{u^{n}}-\sum_{k=0}^{n-1} \frac{f^{(k)}(0)}{u^{n-k}} .
$$

Proof. By definition, the Laplace transform for $f^{(n)}(t)$ is given by

$$
F_{n}(s)=s^{n} F(u)-\sum_{k=0}^{n-1} s^{n-(k+1)} f^{(k)}(0) .
$$

Therefore,

$$
F_{n}\left(\frac{1}{u}\right)=\frac{F(1 / u)}{u^{n}}-\sum_{k=0}^{n-1} \frac{f^{(k)}(0)}{u^{n-(k+1)}} .
$$

Now, since $G_{k}(u)=F_{k}(1 / u) / u$, for $0 \leq k \leq m$, we have

$$
G_{n}(u)=\frac{G(u)}{u^{n}}-\sum_{k=0}^{n-1} \frac{f^{(k)}(0)}{u^{n-k}}=\frac{1}{u^{n}}\left[G(u)-\sum_{k=0}^{n-1} u^{k} f^{(k)}(0)\right] .
$$

In particular, this means that the Sumudu transform of the second derivative of the function $f$ is given by

$$
G_{2}(u)=\mathbb{S}\left(f^{\prime \prime}(t)\right)=\frac{G(u)}{u^{2}}-\frac{f(0)}{u^{2}}-\frac{f^{\prime}(0)}{u} .
$$

For instance, applying (2.12) to the function $\sin t$ leads us to the equation

$$
-G(u)=\frac{G(u)}{u^{2}}-\frac{1}{u}
$$

whose solution is obviously the Sumudu transform of $\sin t$, given by (2.2).

Theorem 2.3. Let $G^{1}(u)$ and $F^{1}(s)$ denote the Sumudu and the Laplace transforms of the definite integral of $f, W(t)=\int_{0}^{t} f(\tau) d \tau$, respectively. Then

$$
G^{1}(u)=\mathbb{S}(W(t))=u G(u) .
$$

Proof. By definition, the Laplace transform of $W(t)=\int_{0}^{t} f(\tau) d \tau$ is given by

$$
F^{1}(s)=\mathfrak{E}(W(t))=\frac{F(s)}{s} .
$$

Hence,

$$
G^{1}(u)=\frac{F^{1}(1 / u)}{u}=\frac{u F(1 / u)}{u}=F\left(\frac{1}{u}\right)=u G(u)
$$


Now, recall that the antiderivative of the Dirac delta function, $\delta(t-a)$ (see, e.g., [4]), is the Heaviside function $H(t-a)$ defined by

$$
H(t-a)= \begin{cases}0, & \text { if } t<a \\ 1, & \text { if } t>a .\end{cases}
$$

Knowing that the Sumudu transform of $\delta(t-a)$ is $e^{-a / u} / u$ (see entries 34 and 35 in Table A.1), using (2.14), yields the transform of $H(t-a)$ :

$$
\mathbb{S}[H(t-a)]=e^{-a / u} .
$$

Another facet of the duality relation between this transform and the Laplace transform is revealed through the interchange of the images of $H(t)$ and $\delta(t)$.

Note that the Sumudu transforms of $H(t)$ and $\delta(t)$ are 1 and $1 / u$, respectively. This is consistent with the units and the buildup of these functions (see, e.g., [4, Section 6.4]). Now, the $\operatorname{Dirac} \delta(t)$ is really a generalized function. Thus, recalling (2.14), we may be able to make sense, in a generalized fashion, of the notion of a derivative for $\delta(t)$. Indeed, if a generalized function $g(t)$ were to exist such that

$$
\mathbb{S}\left(\int_{0}^{t} g(\tau) d \tau\right)=\frac{1}{u}
$$

then, we must have

$$
\int_{0}^{t} g(\tau) d \tau=\delta(t)
$$

This result will be useful in Section 4. Next we establish the scale-preserving property of this transform.

Theorem 2.4. Let $f(t) \in A$ with Laplace and Sumudu transforms $F(s)$ and $G(u)$, respectively. Then

$$
\mathbb{S}(f(a t))=G(a u)
$$

Proof. The Sumudu transform of $f(a t)$ may be obtained directly from the definition (1.2):

$$
\mathbb{S}(f(a t))=\int_{0}^{\infty} f(a u t) e^{-t} d t=G(a u)
$$

or via the working defining equation (2.1). Indeed, since (see, e.g., [4])

$$
\mathfrak{L}(f(a t))=\frac{1}{a} F\left(\frac{s}{a}\right)
$$

then

$$
\mathbb{S}(f(a t))=\frac{(1 / a) F(1 / a u)}{u}=\frac{F(1 / a u)}{a u}=G(a u)
$$


Theorem 2.5. Let $f \in A$ with Sumudu transform $G(u)$. Then,

$$
\mathbb{S}\left(t \frac{d f(t)}{d t}\right)=u \frac{d G(u)}{d u}
$$

Proof. From the defining equation (1.1),

$$
\frac{d G(u)}{d u}=\frac{d}{d u} \int_{0}^{\infty} f(u t) e^{-t} d t=\int_{0}^{\infty} \frac{d}{d u} f(u t) e^{-t} d t=\int_{0}^{\infty} t e^{-t} \frac{d f(u t)}{d t} d t
$$

Hence

$$
\frac{d G(u)}{d u}=\frac{1}{u} \int_{0}^{\infty}(u t) f^{\prime}(u t) e^{-t} d t=\frac{1}{u} \mathbb{S}\left(t \frac{d f(t)}{d t}\right)
$$

Multiplying both sides by $u$, we get the desired result in (2.25).

We observe that with the Sumudu transform, differentiation and integration in the $t$-domain are akin to division and multiplication in the $u$-domain, respectively. Furthermore, many of the scaling properties of $f(t)$ are carried over to its Sumudu transform $G(u)$. Hence both $f(t)$ and $G(u)$ keep the same units, and $u$ and $G(u)$ can be treated as replicas of $t$ and $f(t)$, respectively. This is a major advantage in transform theory, especially when dealing with applications, where being aware of the units of the quantities described as well as the dimensionless factor groups may be extremely relevant in problem solving.

In view of these advantages and the duality relation between the Sumudu and Laplace transforms, there may be applied situations where using the Sumudu transform may be favored over using the Laplace transform. This will be illustrated at length in an upcoming paper dealing with Brownian motion and weighted convection diffusion equations (see $[1,2])$. In the meantime, we take advantage of the duality to investigate more properties of the Sumudu transform.

\section{More properties of the Sumudu transform}

The next few theorems establish some translation, shift, and limit properties of the Sumudu transform.

Theorem 3.1. Let $f(t) \in A$ with Sumudu transform $G(u)$. Then,

$$
\mathbb{S}\left(e^{a t} f(t)\right)=\frac{1}{1-a u} G\left(\frac{u}{1-a u}\right) .
$$

Proof. From (1.2), we see that

$$
\mathbb{S}\left(e^{a t} f(t)\right)=\int_{0}^{\infty} f(u t) e^{-(1-a u) t} d t
$$


110 The Sumudu transform

Therefore, by a change of variable $(w=(1-a u) t)$, we get that

$$
\mathbb{S}\left(e^{a t} f(t)\right)=\frac{1}{1-a u} \int_{0}^{\infty} f\left(\frac{u w}{1-a u}\right) e^{-w} d w=\frac{1}{1-a u} G\left(\frac{u}{1-a u}\right) .
$$

Theorem 3.2. Let $f(t) \in A$ with Laplace and Sumudu transforms $F(s)$ and $G(u)$, respectively. Then the function

$$
h(t)= \begin{cases}f(t-a), & \text { if } t>a, \\ 0, & \text { if } t<a,\end{cases}
$$

has a Sumudu transform given by

$$
\mathbb{S}(h(t))=e^{-a / u} G(u) .
$$

Proof. Note that from (2.17), $h(t)=H(t-a) f(t-a)$, and hence the Laplace transform of $h(t)$ is given by

$$
\mathfrak{E}(h(t))=e^{-a s} F(s) .
$$

Therefore, by duality, the Sumudu transform of $h(t)$ is given by

$$
\mathbb{S}(h(t))=e^{-a / u} \frac{F(1 / u)}{u}=e^{-a / u} G(u) .
$$

The next theorem shows that the average of $f$ over $[0, t]$ transforms to the average of G over $[0, u]$.

Theorem 3.3. Let $f(t) \in A$ with Sumudu transform $G(u)$. Then,

$$
\mathbb{S}\left(\frac{1}{t} \int_{0}^{t} f(\tau) d \tau\right)=\frac{1}{u} \int_{0}^{u} G(v) d v
$$

Proof. From definition (1.1), we have

$$
\begin{aligned}
\frac{1}{u} \int_{0}^{u} G(v) d v & =\frac{1}{u} \int_{0}^{u} \int_{0}^{\infty} f(v t) e^{-t} d t d v=\frac{1}{u} \int_{0}^{\infty} e^{-t} \int_{0}^{\infty} f(v t) d v d t \\
& =\int_{0}^{\infty} \frac{e^{-t}}{u} \int_{0}^{u} f(w) \frac{d w}{t} d t=\int_{0}^{\infty} \frac{1}{u t} e^{-t} \int_{0}^{u t} f(w) d w d t \\
& =\int_{0}^{\infty} \frac{1}{u t}\left[\int f(w) d w\right]_{0}^{u t} e^{-t} d t=\mathbb{S}\left(\frac{1}{t} \int_{0}^{t} f(\tau) d \tau\right)
\end{aligned}
$$

Theorem 3.4. Let $f(t) \in A$ and suppose that either $\lim _{t \rightarrow 0} f(t)$ or $\lim _{t \rightarrow \infty} f(t)$ exists. Then

$$
\begin{aligned}
\lim _{u \rightarrow 0} G(u) & =\lim _{t \rightarrow 0} f(t), \\
\lim _{u \rightarrow \infty} G(u) & =\lim _{t \rightarrow \infty} f(t) .
\end{aligned}
$$


Proof. The first limit is obtained as follows:

$$
\begin{aligned}
\lim _{u \rightarrow 0} G(u) & =\lim _{u \rightarrow 0} \int_{0}^{\infty} f(u t) e^{-t} d t=\int_{0}^{\infty}\left[\lim _{u \rightarrow 0} f(u t)\right] e^{-t} d t \\
& =\int_{0}^{\infty}\left[\lim _{w \rightarrow 0} f(w)\right] e^{-t} d t=\lim _{w \rightarrow 0} f(w) \int_{0}^{\infty} e^{-t} d t=\lim _{w \rightarrow 0} f(w) .
\end{aligned}
$$

In the same manner,

$$
\begin{aligned}
\lim _{u \rightarrow \infty} G(u) & =\int_{0}^{\infty}\left[\lim _{u \rightarrow \infty} f(u t)\right] e^{-t} d t \\
& =\int_{0}^{\infty}\left[\lim _{w \rightarrow \infty} f(w)\right] e^{-t} d t=\lim _{w \rightarrow \infty} f(w) .
\end{aligned}
$$

A similar argument yields the negative counterpart to (3.11):

$$
\lim _{u \rightarrow-\infty} G(u)=\lim _{t \rightarrow-\infty} f(t)
$$

when the right-hand side of (3.14) exists.

The results indicated in (3.10) and (3.11) are known to be the initial and final value theorems, respectively. The reader can observe that most of the previous proofs may also be obtained by the duality relation (2.1). For instance, (3.10) and (3.11) can alternatively be gotten as follows:

$$
\lim _{u \rightarrow 0} G(u)=\lim _{u \rightarrow 0} \frac{F(1 / u)}{u}=\lim _{s \rightarrow \infty} s F(s)=\lim _{t \rightarrow 0} f(t) .
$$

Similarly,

$$
\lim _{u \rightarrow \infty} G(u)=\lim _{s \rightarrow 0} s F(s)=\lim _{t \rightarrow \infty} f(t) .
$$

We conclude this section by establishing a formulation for the Sumudu transform of periodic functions.

Theorem 3.5. Let $f(t) \in A$ be T-periodic. Then the Sumudu transform of $f(t)$ is given by

$$
\mathbb{S}(f(t))=\frac{\int_{0}^{T / u} e^{-t} f(u t) d t}{1-e^{-T / u}}
$$

Proof. The Laplace transform of the periodic function $f(t)$ is given by

$$
\mathfrak{L}(f(t))=\frac{\int_{0}^{T} e^{-s t} f(t) d t}{1-e^{-s T}} .
$$

Note that

$$
\int_{0}^{T} e^{-s t} f(t) d t=u \int_{0}^{T / u} e^{-t} f(u t) d t
$$


and that, in particular,

$$
1-e^{-T / u}=\int_{0}^{T / u} e^{-t} d t
$$

Therefore, by definition (2.1), we have

$$
G(u)=\frac{1}{u}[\mathfrak{L}(f(t))]_{s=1 / u}=\frac{\int_{0}^{T} e^{-t / u} f(t) d t}{u\left[1-e^{-T / u}\right]}=\frac{\int_{0}^{T / u} e^{-t} f(u t) d t}{1-e^{-T / u}} .
$$

\section{Applications to an integral production problem}

In this section, we use the Sumudu transform to treat a production-depreciation problem, first considered by Kalla et al. [3]. This problem examines the manner the production of an item varies in time, when for known losses due to depreciation, the total amount of the product is to remain constant. The following model can easily be adopted to weighted population-growth models (see [1]), birth-death processes, and hormonal and drug release control problems.

If at $t=0$, the unused amount of a product is $M$, despite the exposure of the amount $M$ of the product to a depreciation function $f(t)$, for $t \geq 0$, we like the production of this item $g(t)$ to counterbalance the depreciation so as to keep the amount $M$ at all times. Note that there is no loss of generality in assuming that the depreciation function over time satisfies the condition

$$
\int_{0}^{\infty} f(t) d t=1
$$

Hence, the amount lost due depreciation in the absence of production $(g(t)=0, t>0)$ is given by

$$
\int_{0}^{\infty} M f(t) d t=M
$$

The amount of the item produced in the absence of depreciation $(f(t) \equiv 0)$ in an interval of time $[x, x+\Delta x]$ is given by $g(x) \Delta x$. When depreciation takes place, the amount of the item lost at a later time $t$ is given by $g(x) f(t-x) \Delta x$, and the total loss due to depreciation from the start until time $t$ is given by the convolution integral

$$
(f * g)(t)=\int_{0}^{t} g(x) f(t-x) d x
$$

Therefore, if both production and depreciation are simultaneously in effect by the time $t$, the net difference of production and loss must equal $M f(t)$. That is,

$$
\int_{0}^{t} g(x) d x-\int_{0}^{t} f(t-x) g(x) d x=M f(t)
$$


Kalla et al. [3] solved (4.4) for the depreciation function

$$
f(t)=\frac{(a)^{k}}{\Gamma(k)} t^{k-1} e^{-a t}
$$

with constants $a$ and $k$ (see entry 10 in Table A.1), and obtained the production function

$$
g(t)=M \sum_{n=1}^{\infty} \frac{n ! a^{n k}}{\Gamma(n k)} t^{n k-n-1} e^{-a t} L_{n}^{n k-n-1}(-a t),
$$

where $L_{n}^{p}(x)$ are the generalized Laguerre polynomials.

To solve the integral equation (4.4) our way, we first introduce the Sumudu transform convolution theorem.

Theorem 4.1. Let $f(t)$ and $g(t)$ be in $A$, having Laplace transforms $F(s)$ and $G(s)$, respectively, and Sumudu transforms $M(u)$ and $N(u)$, respectively. Then the Sumudu transform of the convolution of $f$ and $g$,

$$
(f * g)(t)=\int_{0}^{\infty} f(t) g(t-\tau) d \tau
$$

is given by

$$
\mathbb{S}((f * g)(t))=u M(u) N(u)
$$

Proof. First, recall that the Laplace transform of $(f * g)$ is given by

$$
\mathfrak{E}((f * g) t)=F(s) G(s) .
$$

Now, since, by the duality relation,

$$
\mathbb{S}((f * g)(t))=\frac{1}{u} \mathfrak{E}((f * g)(t))
$$

and since

$$
M(u)=\frac{F(1 / u)}{u}, \quad N(u)=\frac{G(1 / u)}{u},
$$

the Sumudu transform of $(f * g)$ is obtained as follows:

$$
\begin{aligned}
\mathbb{S}((f * g)(t)) & =\frac{F(1 / u) \times G(1 / u)}{u} \\
& =u \frac{F(1 / u)}{u} \frac{G(1 / u)}{u} \\
& =u M(u) N(u) .
\end{aligned}
$$




\section{The Sumudu transform}

Now, to solve our production problem, letting $G(u)$ and $F(u)$ be Sumudu transforms of the sought production function $g(t)$ and of the depreciation function $f(t)$, respectively, (4.4) becomes

$$
u G(u)-u G(u) F(u)=M F(u)
$$

Therefore, we have

$$
G(u)=\frac{M F(u)}{u[1-F(u)]}
$$

Let $f(t)=e^{-t}$, then

$$
\begin{gathered}
F(u)=\frac{1}{1+u}, \\
G(u)=\frac{M(1 /(1+u))}{u[1-1 /(1+u)]}=\frac{M}{u^{2}} .
\end{gathered}
$$

Consequently, from (4.16),

$$
\frac{M}{u}=u G(u)=\mathbb{S}\left(\int_{0}^{t} g(\tau) d \tau\right)
$$

Therefore, by linearity of the Sumudu transform (2.19) and (2.20), we deduce that

$$
\int_{0}^{t} g(\tau) d \tau=M \delta(t)
$$

Now, in light of (4.5) and (4.6), our depreciation function coincides with that of Kalla et al. [3], when $a=k=1$. Hence, our solution $g(t)$ must agree with theirs for the same values. For $f(t)=e^{-t}$, we take the production function

$$
g(t)=\frac{e^{-t}}{t} \sum_{n=1}^{\infty} n L_{n}^{-1}(-t),
$$

where $L_{n}^{-1}$ are the Laguerre generalized polynomials. On the other hand, $g(t)$ is to satisfy (4.18). Therefore, we must have

$$
\delta(t)=\sum_{n=1}^{\infty} n \int_{0}^{t} \frac{e^{-\tau}}{\tau} L_{n}^{-1}(-\tau) d \tau
$$

Whence, if there is a notion of a generalized derivative for $\delta(t)$, then $g(t)$, as defined in (4.19), is a most likely suspect. 
Fethi Bin Muhammed Belgacem et al. 115

\section{Appendix}

Table A.1. Laplace and Sumudu transforms of some functions.

\begin{tabular}{|c|c|c|c|}
\hline & $f(t)$ & $F(s)=\mathfrak{E}\{f(t)\}$ & $G(u)=\mathbb{S}(f(t))$ \\
\hline \multirow[t]{2}{*}{1} & \multirow[t]{2}{*}{1} & $\frac{1}{-}$ & \multirow[t]{2}{*}{1} \\
\hline & & $\begin{array}{l}s \\
1 \\
\end{array}$ & \\
\hline 2 & $t$ & $\overline{s^{2}}$ & $u$ \\
\hline 3 & $\frac{t^{n-1}}{(n-1) !}, n=1,2, \ldots$ & $\frac{1}{c^{n}}$ & $u^{n-1}$ \\
\hline \multirow{2}{*}{4} & $\begin{array}{l}(n-1) ! \\
1\end{array}$ & $\begin{array}{r}s^{n} \\
1\end{array}$ & 1 \\
\hline & $\overline{\sqrt{\pi t}}$ & $\overline{\sqrt{s}}$ & $\sqrt{\sqrt{u}}$ \\
\hline \multirow[t]{2}{*}{5} & $2 \sqrt{\frac{t}{-}}$ & 1 & \multirow{2}{*}{$\sqrt{u}$} \\
\hline & $t^{a-1}$ & $s^{3 / 2}$ & \\
\hline 6 & $\overline{\Gamma(a)}, a>0$ & $\frac{1}{s^{a}}$ & $u^{a-1}$ \\
\hline \multirow[t]{2}{*}{7} & \multirow{2}{*}{$e^{a t}$} & 1 & 1 \\
\hline & & $\begin{array}{r}s-a \\
1\end{array}$ & $1-a u$ \\
\hline 8 & $t e^{a t}$ & $\overline{(s-a)^{2}}$ & $\overline{(1-a u)^{2}}$ \\
\hline \multirow[t]{2}{*}{9} & $\frac{1}{(n-1) !} t^{n-1} e^{a t}, n=1,2, \ldots$ & 1 & $u^{n-1}$ \\
\hline & \multirow{3}{*}{$\begin{array}{l}\frac{1}{\Gamma(k)} t^{k-1} e^{a t}, k>0 \\
\frac{1}{(a-b)}\left(e^{a t}-e^{b t}\right), a \neq b\end{array}$} & $\begin{array}{c}(s-a)^{n} \\
1\end{array}$ & $\begin{array}{c}(1-a u)^{n} \\
u^{k-1}\end{array}$ \\
\hline 10 & & $\overline{(s-a)^{k}}$ & $\overline{(1-a u)^{k}}$ \\
\hline 11 & & $\frac{1}{(s-a)(s-b)}$ & $\frac{u}{(1-a u)(1-b u)}$ \\
\hline 12 & $\frac{1}{(a-b)}\left(a e^{a t}-b e^{b t}\right), a \neq b$ & $\frac{s}{(s-a)(s-b)}$ & $\frac{1}{(1-a u)(1-b u)}$ \\
\hline 13 & $\frac{1}{\omega} \sin \omega t$ & $\frac{1}{s^{2}+\omega^{2}}$ & $\frac{u}{1+\omega^{2} u^{2}}$ \\
\hline 14 & $\cos \omega t$ & $\frac{s}{s^{2}+\omega^{2}}$ & $\frac{1}{1+\omega^{2} u^{2}}$ \\
\hline 15 & $\frac{1}{a} \sinh a t$ & $\frac{1}{s^{2}-a^{2}}$ & $\frac{u}{1-a^{2} u^{2}}$ \\
\hline 16 & $\cosh a t$ & $\frac{s}{s^{2}-a^{2}}$ & $\frac{1}{1-a^{2} u^{2}}$ \\
\hline 17 & $\frac{1}{\omega} e^{a t} \sin \omega t$ & $\frac{1}{(s-a)^{2}+\omega^{2}}$ & $\frac{u}{(1-a u)^{2}+\omega^{2} u^{2}}$ \\
\hline 18 & $e^{a t} \cos \omega t$ & $\frac{s-a}{(s-a)^{2}+\omega^{2}}$ & $\frac{1-a u}{(1-a u)^{2}+\omega^{2} u^{2}}$ \\
\hline 19 & $\frac{1}{\omega^{2}}(1-\cos \omega t)$ & $\frac{1}{s\left(s^{2}+\omega^{2}\right)}$ & $\frac{u^{2}}{1+\omega^{2} u^{2}}$ \\
\hline 20 & $\frac{1}{\omega^{3}}(\omega t-\sin \omega t)$ & $\frac{1}{s^{2}\left(s^{2}+\omega^{2}\right)}$ & $\frac{u^{3}}{1+\omega^{2} u^{2}}$ \\
\hline 21 & $\frac{1}{2 \omega^{3}}(\sin \omega t-\omega t \cos \omega t)$ & $\frac{1}{\left(s^{2}+\omega^{2}\right)^{2}}$ & $\frac{u^{3}}{\left(1+\omega^{2} u^{2}\right)^{2}}$ \\
\hline 22 & $\frac{t}{2 \omega} \sin \omega t$ & $\frac{s}{\left(s^{2}+\omega^{2}\right)^{2}}$ & $\frac{u^{2}}{\left(1+\omega^{2} u^{2}\right)^{2}}$ \\
\hline
\end{tabular}


116 The Sumudu transform

Table A.1. Continued.

\begin{tabular}{|c|c|c|c|}
\hline & $f(t)$ & $F(s)=\mathfrak{E}\{f(t)\}$ & $G(u)=\mathbb{S}(f(t))$ \\
\hline 23 & $\frac{1}{2 \omega}(\sin \omega t+\omega t \cos \omega t)$ & $\frac{s^{2}}{\left(s^{2}+\omega^{2}\right)^{2}}$ & $\frac{u}{\left(1+\omega^{2} u^{2}\right)^{2}}$ \\
\hline 24 & $\frac{1}{b^{2}-a^{2}}(\cos a t-\cos b t),\left(a^{2} \neq b^{2}\right)$ & $\frac{s}{\left(s^{2}+a^{2}\right)\left(s^{2}+b^{2}\right)}$ & $\frac{u^{2}}{\left(1+a^{2} u^{2}\right)\left(1+b^{2} u^{2}\right)}$ \\
\hline 25 & $\frac{1}{4 k^{3}}(\sin k t \cosh k t-\cos k t \sinh k t)$ & $\frac{1}{s^{4}+4 k^{4}}$ & $\frac{u^{3}}{1+4 k^{4} u^{4}}$ \\
\hline 26 & $\frac{1}{2 k^{2}} \sin k t \sinh k t$ & $\frac{s}{s^{4}+4 k^{4}}$ & $\frac{u^{2}}{1+4 k^{4} u^{4}}$ \\
\hline 27 & $\frac{1}{2 k^{3}}(\sinh k t-\sin k t)$ & $\frac{1}{s^{4}-k^{4}}$ & $\frac{u^{3}}{1-k^{4} u^{4}}$ \\
\hline 28 & $\frac{1}{2 k^{2}}(\cosh k t-\cos k t)$ & $\frac{s}{s^{4}-k^{4}}$ & $\frac{u^{2}}{1-k^{4} u^{4}}$ \\
\hline 29 & $\frac{1}{2 \sqrt{\pi t^{3}}}\left(e^{b t}-e^{a t}\right)$ & $\sqrt{s-a}-\sqrt{s-b}$ & $\frac{\sqrt{1-a u}-\sqrt{1-b u}}{u^{3 / 2}}$ \\
\hline 30 & $e^{-(a+b) t / 2} I_{0}\left(\frac{a-b}{2} t\right)$ & $\frac{1}{\sqrt{s+a} \sqrt{s+b}}$ & $\frac{1}{\sqrt{1+a u} \sqrt{1+b u}}$ \\
\hline 31 & $J_{0}(a t)$ & $\sqrt{\sqrt{s^{2}+a^{2}}}$ & $\frac{1}{\sqrt{1+a u^{2}}}$ \\
\hline 32 & $\frac{1}{\sqrt{\pi t}} e^{a t}(1+2 a t)$ & $\frac{s}{(s-a)^{3 / 2}}$ & $\frac{u^{3}}{u^{1 / 2}(1-a u)^{3 / 2}}$ \\
\hline 33 & $\frac{\sqrt{\pi}}{\Gamma(k)}\left(\frac{t}{2 a}\right)^{k-1 / 2} I_{k-1 / 2}(a t),(k>0)$ & $\frac{1}{\left(s^{2}-a^{2}\right)^{k}}$ & $\frac{u^{2 k-1}}{\left(1-a^{2} u^{2}\right)^{k}}$ \\
\hline 34 & $H(t-a)$ & $\frac{1}{s} e^{-a s}$ & $e^{-a / u}$ \\
\hline 35 & $\delta(t-a)$ & $e^{-a s}$ & $\frac{1}{u} e^{-a / u}$ \\
\hline 36 & $J_{0}(2 \sqrt{k t})$ & $\frac{1}{s} e^{-k / s}$ & $e^{-k u}$ \\
\hline 37 & $\frac{1}{\sqrt{\pi t}} \cos 2 \sqrt{k t}$ & $\frac{1}{\sqrt{s}} e^{-k / s}$ & $\frac{1}{\sqrt{u}} e^{-k u}$ \\
\hline 38 & $\frac{1}{\sqrt{\pi t}} \sinh 2 \sqrt{k t}$ & $\frac{1}{s^{3 / 2}} e^{k / s}$ & $\sqrt{u} e^{k u}$ \\
\hline 39 & $\frac{k}{2 \sqrt{\pi t^{3}}} e^{-k^{2} / 4 t},(k>0)$ & $e^{-k \sqrt{s}}$ & $\frac{1}{u} e^{-k / \sqrt{u}}$ \\
\hline 40 & $\ln t+\gamma(\gamma \simeq 0.5772 \ldots)$ & $-\frac{1}{s} \ln s$ & $\ln u$ \\
\hline 41 & $\frac{1}{t}\left(e^{b t}-e^{a t}\right)$ & $\ln \frac{s-a}{s-b}$ & $\frac{1}{u} \ln \frac{1-a u}{1-b u}$ \\
\hline 42 & $\frac{2}{t}(1-\cos \omega t)$ & $\ln \frac{s^{2}+\omega^{2}}{s^{2}}$ & $\frac{1}{u} \ln \left(1+\omega^{2} u^{2}\right)$ \\
\hline 43 & $\frac{2}{t}(1-\cosh a t)$ & $\ln \frac{s^{2}-a^{2}}{s^{2}}$ & $\frac{1}{u} \ln \left(1-a^{2} u^{2}\right)$ \\
\hline 44 & $\frac{1}{t} \sin \omega t$ & $\arctan \frac{\omega}{s}$ & $\frac{1}{u} \arctan \omega u$ \\
\hline 45 & $\operatorname{Si}(t)$ & $\frac{1}{s} \operatorname{arccot} s$ & func $\operatorname{arccot} \frac{1}{u}$ \\
\hline
\end{tabular}


Table A.2. The Sumudu transform properties.

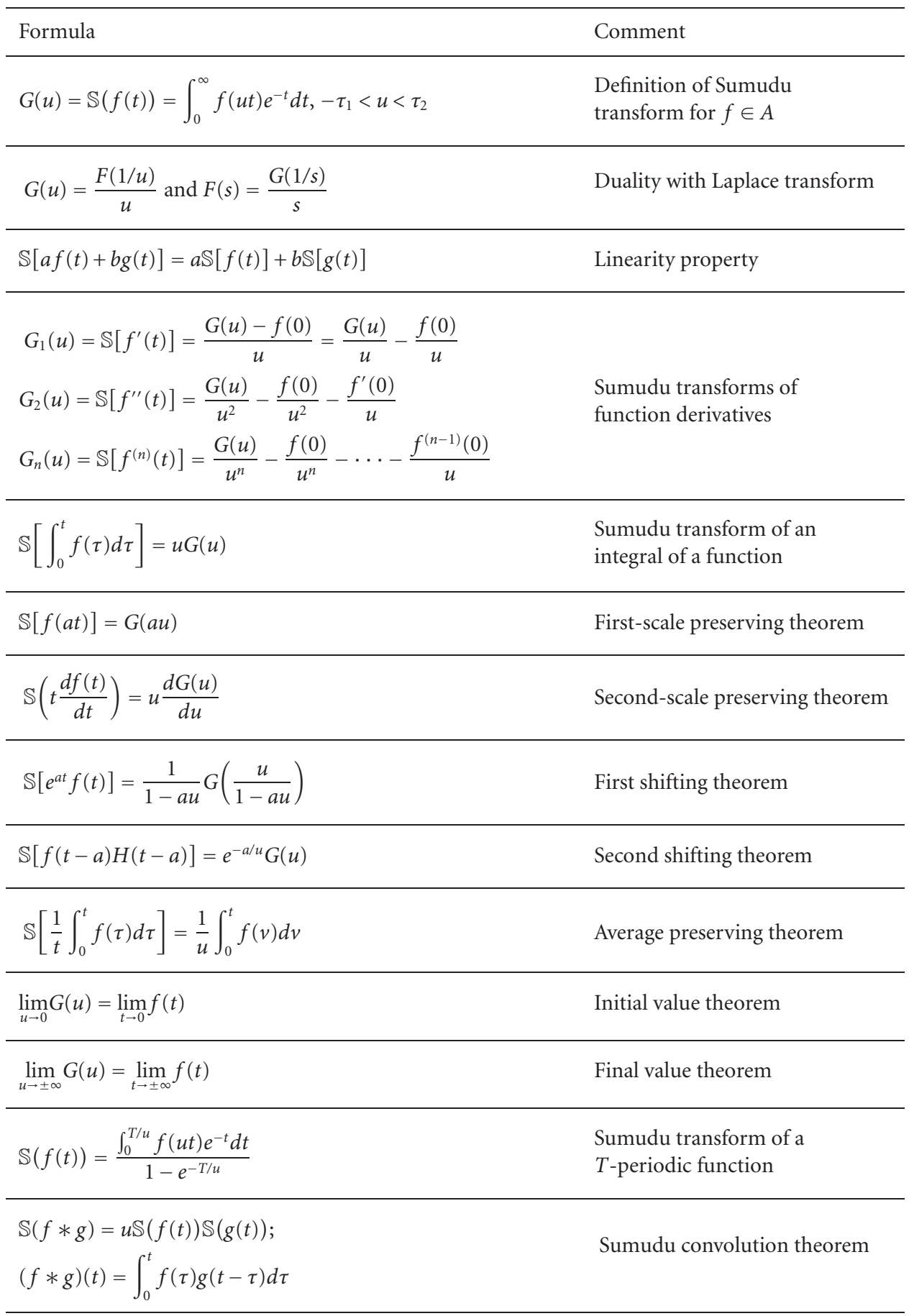




\section{The Sumudu transform}

\section{References}

[1] F. Belgacem, Elliptic Boundary Value Problems with Indefinite Weights: Variational Formulations of the Principal Eigenvalue and Applications, Pitman Research Notes in Mathematics Series, vol. 368, Longman, Harlow, 1997.

[2] Diffusion and drift models for population dispersal from stochastic and continuum views, Int. J. Appl. Math. 5 (2001), no. 1, 85-106.

[3] S. L. Kalla, L. Viloria, and S. Conde, On an integral equation associated with a production problem, Kyungpook Math. J. 19 (1979), no. 1, 135-147.

[4] E. Kreyszig, Advanced Engineering Mathematics, 7th ed., John Wiley \& Sons, New York, 1993.

[5] N. W. McLachlan, Modern Operational Calculus with Applications in Technical Mathematics, Macmillan, London, 1948.

[6] G. K. Watugala, Sumudu transform - a new integral transform to solve differential equations and control engineering problems, Math. Engrg. Indust. 6 (1998), no. 4, 319-329.

Fethi Bin Muhammed Belgacem: Faculty of Information Technology, Arab Open University, Block 3, Street 7, No. 37, P.O. Box 3322, Safat 13033, Kuwait

E-mail address: fbmbelgacem@yahoo.com

Ahmed Abdullatif Karaballi: Department of Mathematics and Computer Science, Faculty of Science, Kuwait University, P.O. Box 5969, Safat 13060, Kuwait

E-mail address: karabali@mcs.sci.kuniv.edu.kw

Shyam L. Kalla: Department of Mathematics and Computer Science, Faculty of Science, Kuwait University, P.O. Box 5969, Safat 13060, Kuwait

E-mail address: kalla@mcs.sci.kuniv.edu.kw 


\section{Differential Equations \& Nonlinear Mechanics}

\section{An Open Access Journal}

\section{Editor-in-Chief}

K. Vajravelu

USA

Associate Editors

N. Bellomo

Italy

J. L. Bona

USA

J. R. Cannon

USA

S.-N. Chow

USA

B. S. Dandapat

India

E. DiBenedetto

USA

R. Finn

USA

R. L. Fosdick

USA

J. Frehse

Germany

A. Friedman

USA

R. Grimshaw

UK

J. Malek

Czech Republic

J. T. Oden

USA

R. Quintanilla

Spain

K. R. Rajagopal

USA

G. Saccomandi

Italy

Y. Shibata

Japan

Ivar Stakgold

USA

Swaroop Darbha

USA

A. Tani

Japan

S. Turek

Germany

A. Wineman

USA
Website: http://www.hindawi.com/journals/denm/

Aims and Scope

Differential equations play a central role in describing natural phenomena as well as the complex processes that arise from science and technology. Differential Equations \& Nonlinear Mechanics (DENM) will provide a forum for the modeling and analysis of nonlinear phenomena. One of the principal aims of the journal is to promote cross-fertilization between the various subdisciplines of the sciences: physics, chemistry, and biology, as well as various branches of engineering and the medical sciences.

Special efforts will be made to process the papers in a speedy and fair fashion to simultaneously ensure quality and timely publication.

DENM will publish original research papers that are devoted to modeling, analysis, and computational techniques. In addition to original full-length papers, DENM will also publish authoritative and informative review articles devoted to various aspects of ordinary and partial differential equations and their applications to sciences, engineering, and medicine.

\section{Open Access Support}

The Open Access movement is a relatively recent development in academic publishing. It proposes a new business model for academic publishing that enables immediate, worldwide, barrier-free, open access to the full text of research articles for the best interests of the scientific community. All interested readers can read, download, and/or print any Open Access articles without requiring a subscription to the journal in which these articles are published.

In this Open Access model, the publication cost should be covered by the author's institution or research funds. These Open Access charges replace subscription charges and allow the publishers to give the published material away for free to all interested online visitors.

\section{Instructions for Authors}

Original articles are invited and should be submitted through the DENM manuscript tracking system at http://www.mstracking.com/ denm/. Only pdf files are accepted. If, for some reason, submission through the manuscript tracking system is not possible, you can contact denm.support@hindawi.com.

Hindawi Publishing Corporation

410 Park Avenue, 15th Floor, \#287 pmb, New York, NY 10022, USA
HINDAWI 


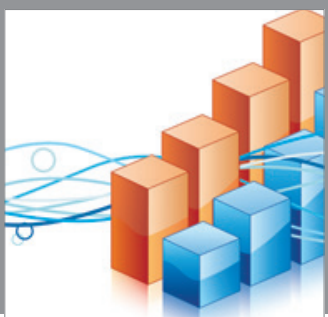

Advances in

Operations Research

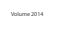

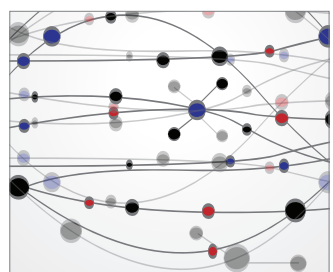

\section{The Scientific} World Journal
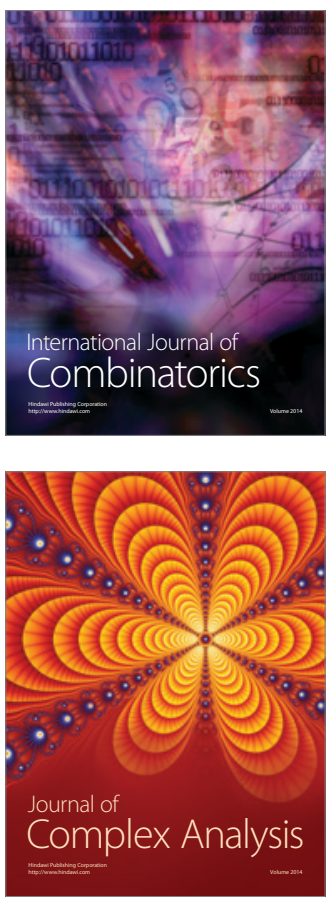

International Journal of

Mathematics and

Mathematical

Sciences
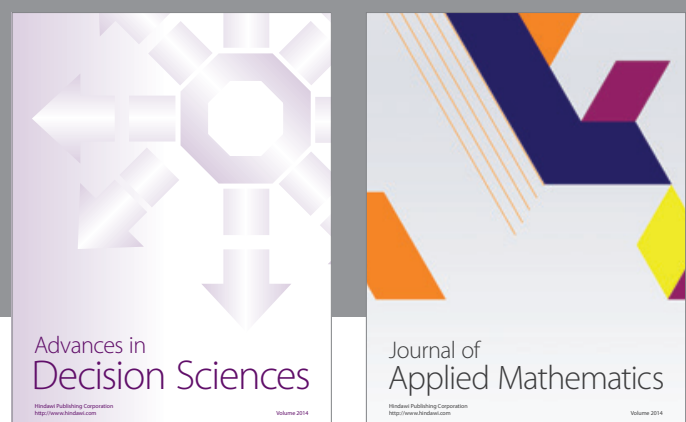

Journal of

Applied Mathematics
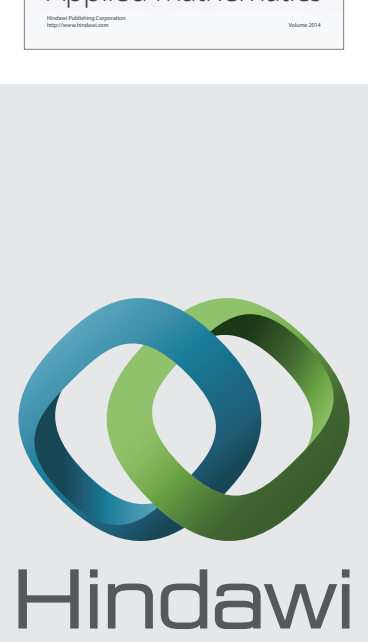

Submit your manuscripts at http://www.hindawi.com
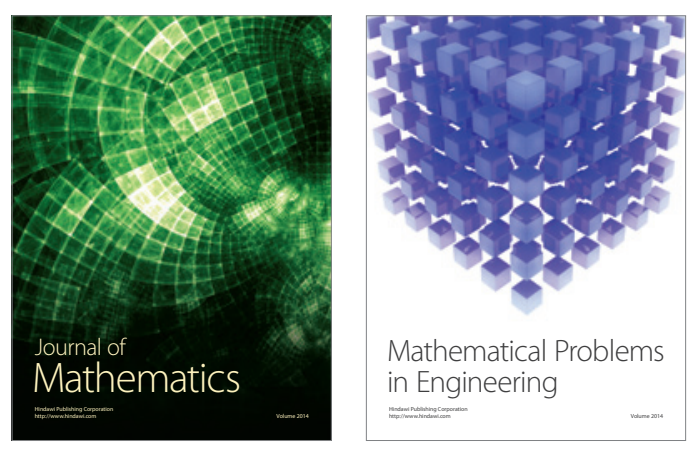

Mathematical Problems in Engineering
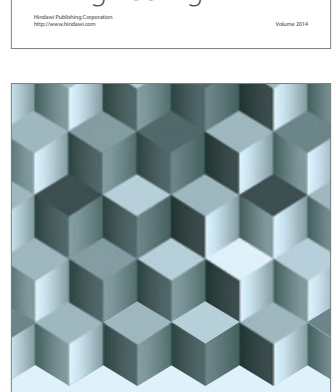

Journal of

Function Spaces
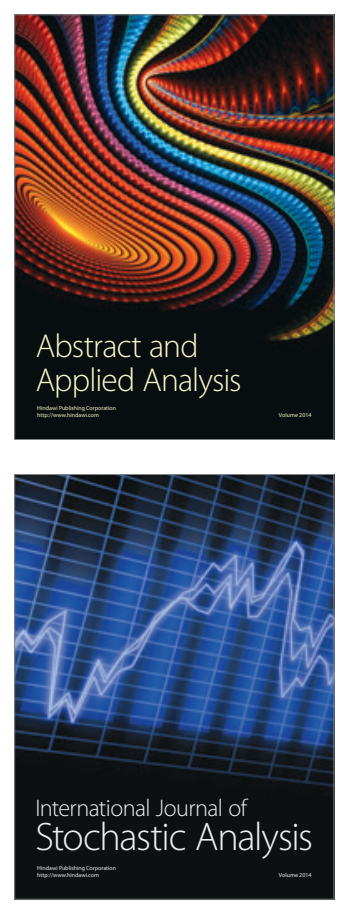

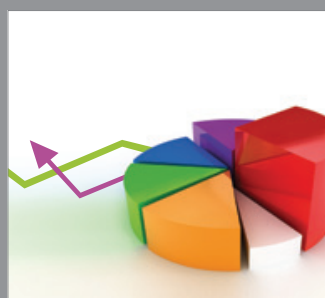

ournal of

Probability and Statistics

Promensencen
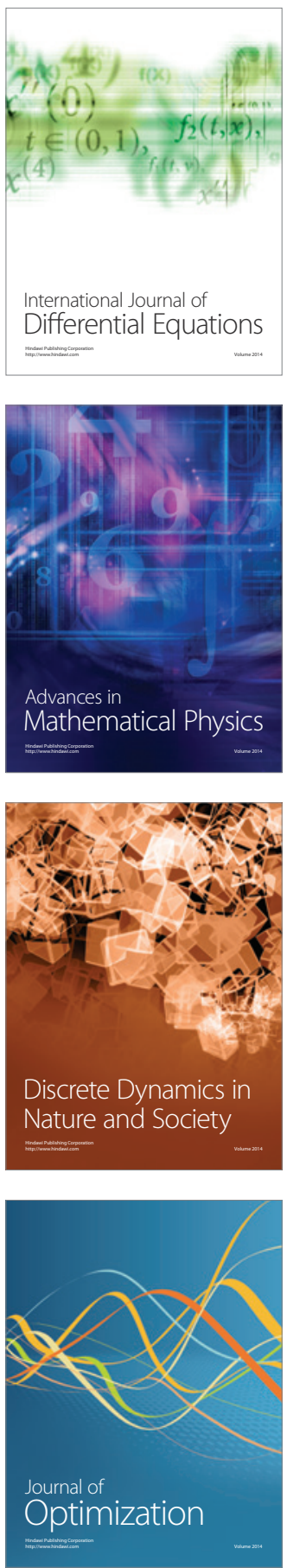